\title{
Depressive symptomatology in pregnant and postpartum women. An exploratory study of the role of maternal antenatal orientations
}

\author{
Johan C. H. van Bussel • Bernard Spitz • \\ Koen Demyttenaere
}

Received: 31 July 2008 /Accepted: 18 February 2009/Published online: 6 March 2009

(C) Springer-Verlag 2009

\begin{abstract}
Little is known about how an expecting woman's view of pregnancy, the child, and motherhood relates to antenatal and postpartum depressive symptomatology. In this study, we investigated the influence of the maternal orientations, as described by Raphael-Leff (Psychological processes of childbearing. The Anna Freud Centre, London, 2005), on the prevalence of depressive symptoms in pregnant and postpartum women. Four hundred three pregnant women participated in a longitudinal study and completed the EPDS and the HADS-D in each pregnancy trimester and between 8 to 12 and 20 to 25 weeks postpartum. In addition, measures of maternal orientation (PPQ), personality (NEO-FFI), coping styles (UCL), adult attachment (RQ), and parental bonding (PBI) were completed antenatally. Bivariate and multivariate analyses revealed that Neuroticism and the Regulator orientation are positively associated with the EPDS and HADS-D in both pregnant and postpartum women. These associations decreased in strength but remained significant after controlling for previous responses on the EPDS and HADS-D. Small negative associations were found between the Facilitator orientation and the HADS-D scores during pregnancy and the early postpartum period. However, this association did not hold its statistical significance within the hierarchical multiple regression models. The maternal orientations have a small but significant and independent contribution in the variance of depressive symptomatology in pregnant and postpartum women.
\end{abstract}

\section{J. C. H. van Bussel ( $₫)$}

Katholieke Universiteit Leuven, Faculty of Medicine,

Institute of Family and Sexuality Studies,

Kapucijnenvoer 33,

3000 Leuven, Belgium

e-mail: johan.vanbussel@med.kuleuven.be

B. Spitz $\cdot$ K. Demyttenaere

Katholieke Universiteit Leuven, Faculty of Medicine,

Leuven, Belgium
Keywords Depressive symptomatology · Pregnancy · Postpartum · Maternal orientation · Personality · Coping . Bonding $\cdot$ Longitudinal

\section{Introduction}

Although common in the transition to motherhood, the prevalence and severity of most common mental health disorders (such as depressive and anxiety disorders) during pregnancy and the postpartum period is not different from a population of non-pregnant or non-postpartum women (van Bussel et al. 2006). However, the mental health condition of pregnant and postpartum women has received considerable clinical and scientific attention because of its suggested influence on the pregnancy, the child, and the family. Indeed, high levels of depressive symptoms in pregnant women have been found to be associated with obstetric outcome (Caesarean section, preeclampsia, spontaneous preterm labour, and birth) (Chung et al. 2001; Dayan et al. 2002, 2006; Kurki et al. 2000; Orr et al. 2002), neonatal outcome (lower birth weight, decrease of vagal tone, admission to neonatal care unit) (Misri et al. 2004; Steer et al. 1992), and behavioural and emotional problems (O'Connor et al. 2002).

Because of these consequences, the identification of factors that determine or trigger elevation of depressive symptomatology in pregnant and postpartum women could contribute to its prevention and treatment. In a recent metaanalysis, both depressive and anxiety disorders during pregnancy and a previous history of depression were identified as the most salient predictors for depressive disorders in the postpartum period (Robertson et al. 2004). Also, intrapersonal variables (Neuroticism, negative life experiences), social variables (marital dissatisfaction, lack of social support), and pregnancy-related clinical variables 
(risk status of current pregnancy, outcome of previous pregnancy) have been associated with higher levels of depressive symptoms in pregnant and postpartum women (Chen et al. 2004; Robertson et al. 2004). Less is known, however, about how an expecting woman's view of pregnancy, the child, and motherhood relates to antenatal and postpartum depressive symptomatology. In their study, Harwood et al. investigated the relation between antenatal parenthood expectations and antenatal depressive symptomatology (Harwood et al. 2007). Contrary to their expectations, they did not find such an association. Despite those results, Harwood et al. (2007) did find that, when motherhood experiences were negative relative to their antenatal expectations, postpartum women reported higher levels of depressive symptoms.

An interesting perspective has also been provided by Raphael-Leff (2005). Based on her clinical experience and qualitative research, she differentiated four maternal orientations or clusters of emotional, cognitive and behavioural expressions of underlying intrapsychic processes related to the pregnancy and motherhood (Raphael-Leff 2005; Sharp and Bramwell 2004). Women tending to the first orientation, called Facilitators, generally feel enriched during their pregnancy and the postpartum period. Spontaneously, they adapt their adult life styles to the world and needs of the dependent foetus or neonatus. A second group of women, the Regulators, fear that motherhood will change their identities and life styles too dramatically. They, therefore, tend more to detachment during pregnancy and to socialization of the newborn child into the own adult world. Most pregnant women, however, positively integrate their ambivalent emotions and uncertainties towards the pregnancy and motherhood (Reciprocators), whereas a small group of women shift between the extreme orientations (Bipolar mothers). According to Raphael-Leff (2005), the factors that elicit heightened depressive symptomatology differ according to these maternal orientations. Women tending to the Regulator orientation are hypothesized to be more vulnerable during the pregnancy and the early postpartum period because they are expected to face a decrease in independency, control, confidence, and social contacts. Women tending more to the Facilitator orientation are hypothesized to be vulnerable in the early postpartum period, when actual motherhood is much less intuitive and evident as expected. Later on, Facilitators are also at risk for heightened levels of depressive symptoms when they need to separate from their children (e.g., to return to employment) and, thus, have to accept that others are also capable of caring for their children. As for the Reciprocators, moderate levels of depressive symptoms during pregnancy and the postpartum period are thought to reflect the growing awareness of their own ambivalent feelings and their realistic appraisal of both the gratifica- tions and responsibilities that come with motherhood (Raphael-Leff 2005).

Partial evidence for these hypotheses was found in both a retrospective survey of a small clinical sample (RaphaelLeff 1985) and a larger prospective study of a representative sample of primiparious women (Sharp and Bramwell 2004). In the latter study, the Regulator orientation contributed significantly to the variance in depressive symptomatology at 6-to-8 weeks postpartum, even after controlling for antenatal depressive symptoms. This latter finding of Sharp and Bramwell (2004) is important because the researchers justly assumed that previous or concurrent depressive responses could affect the completion of the Facilitator and Regulator Questionnaire. In the present study, we further investigated the role of these maternal orientations on the prevalence of depressive symptoms during pregnancy and the postpartum period. Further, the strength of the association between depressive symptomatology and the maternal orientations was compared with that of other intrapsychic variables known for their association with depressive symptomatology (e.g., personality traits, coping styles, adult attachment, and parental bonding) (Demyttenaere et al. 1995; Mickelson et al. 1997; Murphy and Bates 1997; Robertson et al. 2004).

\section{Participants and methods}

\section{Participants}

The present study is part of a longitudinal research project on maternal antenatal and postpartum mental health at the University Hospital Gasthuisberg, Leuven (Belgium). Recruitment of the participants, their background data, and data collection used in this study were described in detail elsewhere (van Bussel et al. 2008) and are summarized here. During 1 year, pregnant women between 8 and 15 gestational weeks were invited to participate in this study during their routine antenatal clinical visit. A nurse or midwife informed the women about the aims and design of the study. Pregnant women with a poor understanding of Dutch, younger than 18 years old, or with a major physical or psychological complication in the pregnancy were not invited to participate in this study. Four hundred and three women, about $20 \%$ of the women who delivered in the hospital during that period, agreed to participate and gave their written consent.

The participants completed a booklet of questionnaires at five time points: between 8 and 15 (T1), 20 and 26 (T2), and 30 and 36 (T3) gestational weeks and between 8 and 12 weeks (T4) and 20 and 25 weeks (T5) postpartum. The first questionnaire booklet was completed at the hospital by 403 women; the following booklets were posted to the 
participants' home addresses. The response rate of the participants in this study was $81.89 \%(n=330)$ at T2, $73.95 \%(n=298)$ at T3, 65.25\% $(n=263)$ at T4, and $50.12 \%$ $(n=202)$ at T5. The most frequent reasons for dropping out were miscarriage, occurrence of a pregnancy complication, and address change.

Ethical approval for this study was received from the Commission on Medical Ethics of the University Hospitals Leuven on 7 May 2005.

\section{Measurements}

The sociodemographic information collected included maternal age, education, marital status, and employment. Furthermore, the participants were asked to report the number of previous pregnancies and miscarriages, whether their current pregnancy was conceived naturally or by reproductive technology, whether their current pregnancy was planned, and how many of their own children they wanted.

\section{Maternal depressive symptoms}

To increase the power of the study, two measures were used to detect depressive symptoms in the participants: the Dutch version (Pop et al. 1992) of the Edinburgh Postnatal Depression Scale (EPDS) (Cox et al. 1987) and the Dutch version (Spinhoven et al. 1997) of the depression scale of the Hospital Anxiety and Depression Scale (HADS-D) (Zigmond and Snaith 1983). The EPDS (ten items) and the HADS-D (seven items) have no physical indicators of psychological distress (headache, weight loss...) which could lead to false positive results due to the pregnancy. Responses on both the EPDS and HADS-D are rated with a four-point ( 0 to 3 ) response category. The EPDS is a widely used measure with good reliability and validity in both a pregnant and postpartum population (Cox and Holden 1994; Murray and Cox 1990). In our study, we found Cronbach's alphas ranging between 0.80 and 0.84 for the EPDS and between 0.75 and 0.84 for the HADS-D. Although the HADS-D was not designed for antenatal or postpartum use, it is widely used and validated within a clinical setting (Herrmann 1997). Also, the HADS-D has no anxiety related symptoms whereas items 4 ("I have been anxious or worried for no good reason") and 5 ("I have felt scared or panicky for no very good reason") of the EPDS are evident anxiety items (Brouwers et al. 2001). The participants completed the EPDS and the HADS-D at every antenatal and postpartum point of measurement (T1-T5).

\section{Intrapsychic independent variables}

To determine women's maternal orientation as defined by Raphael-Leff (2005), the revised Facilitator scale and
Regulator scale of the Placental Paradigm Questionnaire (PPQ) were used (van Bussel et al. 2009). The PPQ is a questionnaire addressing specific antenatal emotional defences (idealization, persecution, obsession, and detachment) towards the self, the child, and the pregnancy. Both scales consist of five items, such as "I feel more of a woman now that I'm pregnant" (Facilitator) and "I feel uneasy about sharing my body with the baby" (Regulator). Responses were rated on a four-point scale. A high score on either the Facilitator scale or the Regulator scale reflected the respondent's tendency to that maternal orientation. Previously, good-to-moderate internal reliabilities were reported for, respectively, the Facilitator scale $(\alpha: 0.75)$ and the Regulator scale $(\alpha: 0.59)$ (van Bussel et al. 2009). At the time this study was conducted, no reliable scales were available for the Reciprocator and Bipolar orientation. The women completed the Facilitator scale and the Regulator scale in the third trimester of their pregnancies.

The Dutch version (Hoekstra et al. 1996) of the NEO Five Factor Inventory (NEO-FFI) (Costa and McCrae 1992) was used to assess general personality traits in the participants. The NEO-FFI (55 items) is a short form of the NEO-Personality Inventory-Revised (NEO-PI-R) (Costa and McCrae 1992), a measure of the Five Factor Model of Personality composed of Neuroticism, Extraversion, Openness to experience, Agreeableness, and Conscientiousness scales. Responses are rated on a five-point scale, ranging from strongly disagree (1) to strongly agree (5). The NEOFFI is a widely used measure with demonstrated good reliability and validity (Murray et al. 2003). Previously, we reported Cronbach's reliability coefficients varying from 0.64 to 0.75 (van Bussel et al. 2008). Participants completed the NEO-FFI in the second pregnancy trimester.

To determine the coping styles of the participants, a Dutch adaptation of the Westbrook Coping Scale was used: the Utrecht Coping List (UCL) (Schreurs and van de Willige 1988). The UCL (47 items) consists of seven scales each addressing a specific coping style: active problem solving, depressive reaction, avoidance, seeking of social support, palliative reaction pattern, expression of negative emotions, and comforting thoughts. Possible answers range from rarely (1) to very often (4). A high score on a scale reflects the frequent use of that coping style. The internal reliabilities and the construct validity reported by Schreurs and van de Willige (1988) are adequate. Previously, we reported Cronbach's reliability coefficients varying from 0.64 to 0.83 (van Bussel et al. 2008). The participants completed the UCL in the second pregnancy trimester.

Retrospective data about the parent-child relation in participants' childhood was obtained with the Dutch version (Verschueren and Marcoen 1993) of the Parental Bonding Inventory (PBI) (Parker et al. 1979). The PBI has 
two versions, a maternal and paternal version, with each having two subscales: "caring" (12 items) and "overprotection" (13 items). Responses are rated on a four-point scale: high scores reflect, respectively, a more affectionate, warm, and caring parent-child interaction and a more dominant and overprotective parent. The PBI is a widely used measure and has a satisfactory construct and convergent validity (Parker 1989). Previously, we reported Cronbach's reliability coefficients varying from 0.83 to 0.92 (van Bussel et al. 2008). Participants completed the PBI in the first pregnancy trimester.

The participants' attachment patterns in close adult peer relationships were determined with the Dutch version (Lowyck et al. 2003) of the Relationship Questionnaire (RQ) (Bartholomew and Horowitz 1991). This measure consists of four short paragraphs, each describing a prototypical attachment pattern (secure, fearful, preoccupied, and dismissing). Participants were asked to rate their degree of correspondence to each attachment pattern on a seven-point scale. The RQ shows convergent validity with interview ratings of adult attachment (Bartholomew and Horowitz 1991; Griffin and Bartholomew 1994). The RQ was completed by the participants in the first pregnancy trimester.

\section{Statistics}

The SAS 9.1 software package was used for statistical analysis (SAS Institute Inc. 2002). Means and standard deviations, absolute numbers and percentages were provided as descriptive statistics. Attrition analyses comparing differences in means of EPDS and HADS-D at T1 of those who participated in all time points of the study $(n=181$, $44.91 \%$ ) and those who dropped out during the study were performed with Student's $t$ tests. Linear mixed-model analyses were performed to observe differences in means of repeated EPDS and HADS-D measurements.

Relations between the EPDS and HADS-D, on the one hand, and the PPQ, PBI, RQ, NEO-FFI, and UCL, on the other, were investigated using Pearson correlations when the responses on the variables were fairly normally distributed; otherwise, Spearman correlations $(\rho)$ were calculated.

To estimate the crude degree of association between the Facilitator scale and Regulator scale, on the one hand, and the EPDS and HADS-D scales, on the other, multiple linear regression analyses were conducted. Due to observed outliers, we used a robust version of the regression procedure, based on Least Trimmed Squares (LTS) estimation (Colin 2006). With this procedure, the weight of the outliers is reduced in favour of the within-range responses.

The Facilitator scale and the Regulator scale were also entered as a final block of independent variables within two series of five hierarchical multiple linear regression (HMLR) analyses. In six steps, demographic variables, parental bonding, adult attachment, personality traits, coping styles, and the Facilitator and Regulator scales were entered in the regression model. The rationale of this order lies in the assumption that parental bonding has a causal influence on both adult attachment (Perris and Andersson 2000), personality (Reti et al. 2002), and coping style (Uehara et al. 1999). Furthermore, a causal relation has been suggested between personality and coping styles (Costa and McCrae 1990; McCrae and Costa 1986). The maternal antenatal orientation scales were added as a final step because we wanted to estimate their additional effect on the other intrapsychic variables. In a secondary analysis, previous EPDS and HADS-D scores were also entered in the HLMR models. To reduce the risk of Type I errors, Bonferroni post-hoc tests were used.

\section{Results}

Most of the participants in this study were Belgian women $(n=390,96.77 \%)$, had a partner $(n=382,95.50 \%)$, had completed higher education $(n=282,70.33 \%)$, and were employed $(n=370,92.04 \%)$. The mean age of the participants was 30.15 years (4.39 SD). One-hundred-forty-nine participants (38.95\%) were primigravida, $101(25.06 \%)$ had had a previous miscarriage, and $42(10.45 \%)$ had conceived after fertility treatment. Most of the pregnancies were planned ( $n=355,88.75 \%)$.

We tested whether the EPDS and HADS-D mean scores at T1 differed among the women in the group who participated at all time points of the study $(n=181$, $44.91 \%)$ and those who dropped out. Both the EPDS ( $t=$ 2.94, $d f=399, p=0.003)$ and HADS-D $(t=2.72, d f=108, p=$ $0.008)$ scores of the dropouts were significantly higher than those of the women who participated at all time points.

The course of depressive symptomatology during pregnancy and the postpartum period

The EPDS mean scores of the participants at T1, T2, T3, T4, and T5 were, respectively: 6.93 (SD 4.44), 6.15 (SD 4.06), 6.35 (SD 4.44), 5.73 (SD 4.19), and 5.08 (SD 4.17). The HADS-D mean scores of the participants at T1, T2, T3, T4, and T5 were, respectively: 3.51 (SD 3.00), 3.15 (SD 2.79), 3.79 (SD 2.89), 3.22 (SD 2.99), and 2.96 (SD 3.19). A linear mixed-model analysis $(F=9.10, d f=1,049, p<$ $0.0001)$ revealed a significant decrease in the EPDS total means in the second pregnancy trimester $(t=2.74, d f=$ $1,049$, adj. $p=0.04)$, between 8 and 12 weeks postpartum $(t=3.65, d f=1,049$, adj. $p=0.003)$, and 20 and 25 weeks postpartum $(t=5.52, d f=1,049$, adj. $p<0.0001)$ as compared 
to the first pregnancy trimester. The EPDS means of the second $(t=3.26, d f=1,049$, adj. $p=0.01)$ and third $(t=4.35$, $d f=1,049$, adj. $p=0.0001)$ pregnancy trimesters were also significantly higher compared to the EPDS mean between 20 and 25 weeks postpartum. A linear mixed-model analysis $(F=5.32, d f=1,045, p=0.0003)$ revealed a significant increase of the HADS-D total mean in the third pregnancy trimester $(t=-3.99, d f=1,045$, adj. $p=0.0007)$ as compared to the second pregnancy trimester. At 8 to 12 weeks postpartum $(t=3.05, d f=1,045$, adj. $p=0.02)$ and 20 to 25 weeks postpartum $(t=3.76, d f=1,045$, adj. $p=$ $0.002)$, the HADS-D total mean decreased as compared to the third pregnancy trimester.

Intrapsychic determinants of depressive symptoms in pregnant and postpartum women

\section{Zero-order correlations}

Zero-order correlations between the antenatal and postpartum maternal scores on the EPDS and HADS-D and the intrapsychic independent variables (PBI, RQ, NEO-FFI, UCL and PPQ) are reported in Table 1. Consistent positive and moderate correlations were found with the Regulator scale, whereas small negative correlations were found with the Facilitator scale (except with the EPDS at T5). Small correlations were also found for the subscales of the PBI, except for T5. More precisely, maternal and paternal overprotection correlated positively with the EPDS and HADS-D in a consistent manner, whereas consistent negative correlations were found with maternal and paternal care, but only with the HADS-D.

Of the adult attachment scales (RQ), the fearful scale and the preoccupied scale correlated consistently with the EPDS scale. Both also correlated positively with the HADS-D at $\mathrm{T} 1$ and $\mathrm{T} 2$. The secure attachment scale correlated negatively with the EPDS and the HADS-D at T1 and T2, but only with the EPDS at T3. No significant correlation was found with dismissing attachment (RQ). Overall, the correlations with the adult attachment scales were small.

Of the personality scales (NEO-FFI), only the Neuroticism scale correlated positively with both the EPDS and the HADS-D in a consistent and strong manner. Small positive correlations were found with the Openness-to-new-experiences scale at all points of measurement, but only with the EPDS. Conversely, small negative correlations were found between the Extraversion scale and the HADS-D scale, except for T5. Small and isolated correlations were found with the Conscientiousness scale, whereas no significant correlation was found with the Agreeableness scale.

Consistent and strong positive correlations were found with the depressive-reaction scale. The positive correlations with the expression-of-negative-emotions scale were also consistent but less strong. Consistent, small negative correlations were found with the active coping scale, but not for T5. Small and isolated correlations were found with the palliative and the avoidance coping scales (both positive) and the seeking-of-social-support scale (negative), whereas no significant correlation was found with the comforting-thoughts scale.

\section{Multiple linear regression analyses}

Multiple linear regression analyses, with both the Facilitator scale and the Regulator scale as independent variables, revealed a significant contribution to the variance on the responses of the EPDS at T1 $\left(F=9.55, p<0.0001, R^{2}=\right.$ $0.06)$, T2 $\left(F=23.11, p<0.0001, R^{2}=0.15\right)$, T3 $(F=25.86$, $\left.p<0.0001, R^{2}=0.16\right)$, T4 $\left(F=15.20, p<0.0001, R^{2}=0.11\right)$, and T5 $\left(F=9.17, p=0.0002, R^{2}=0.09\right)$. Of both orientation scales, only the Regulator scale was significantly associatedin a positive direction - with the EPDS at all points of measurement: $\mathrm{T} 1(\beta=0.18, \mathrm{SE}=0.15, t=3.08, p=0.002)$, T2 $(\beta=0.36, \mathrm{SE}=0.14, t=6.21, p<0.0001), \mathrm{T} 3(\beta=0.35, \mathrm{SE}=$ $0.15, t=6.25, p<0.0001), \mathrm{T} 4(\beta=0.32, \mathrm{SE}=0.15, t=5.01, p<$ $0.0001)$, and T5 $(\beta=0.29, \mathrm{SE}=0.17, t=4.00, p<0.0001)$. The Facilitator scale was significantly associated - in a negative direction - with the EPDS only at $\mathrm{T} 1(\beta=-0.13, \mathrm{SE}=0.09$, $t=-2.23, p=0.03$ ).

A significant contribution of the maternal orientations to the variance on the responses of the HADS-D was found at T1 $\left(F=20.94, p<0.0001, R^{2}=0.13\right)$, T2 $(F=37.59, p<$ $\left.0.0001, R^{2}=0.22\right), \mathrm{T} 3\left(F=41.66, p<0.0001, R^{2}=0.23\right), \mathrm{T} 4$ $\left(F=19.97, p<0.0001, R^{2}=0.15\right)$, and T5 $(F=8.53, p=$ $\left.0.0003, R^{2}=0.09\right)$. The Facilitator scale was significantly associated - in a negative direction - with the HADS-D at $\mathrm{T} 1(\beta=-0.18, \mathrm{SE}=0.05, t=-3.10, p=0.002), \mathrm{T} 2(\beta=-0.27$, $\mathrm{SE}=0.04, t=-4.91, p<0.0001), \mathrm{T} 3(\beta=-0.24, \mathrm{SE}=0.05, t=$ $-4.39, p<0.0001)$, and $\mathrm{T} 4(\beta=-0.17, \mathrm{SE}=0.05, t=-2.78, p=$ $0.006)$. The Regulator scale was significantly associated-in a positive direction - with the HADS-D at T1 $(\beta=0.27, \mathrm{SE}=$ $0.08, t=4.71, p<0.0001), \mathrm{T} 2(\beta=0.32, \mathrm{SE}=0.08, t=5.73, p<$ $0.0001), \mathrm{T} 3(\beta=0.36, \mathrm{SE}=0.09, t=6.66, p<0.0001), \mathrm{T} 4(\beta=$ $0.30, \mathrm{SE}=0.09, t=4.73, p<0.0001)$, and $\mathrm{T} 5(\beta=0.23, \mathrm{SE}=$ $0.10, t=3.05, p=0.003)$.

\section{Hierarchical multiple linear regression (HMLR) analyses}

To estimate the contribution of the maternal orientations above the included intrapsychic variables, hierarchical multiple linear regression analyses were conducted in six steps (see Tables 2 and 3). First, maternal age, primigravidity, and number of previous miscarriages were entered into the HMLR models. Overall, this block of variables significantly explained between $5 \%$ and $10 \%$ of the variation of the EPDS and HADS-D scores at T1 and T2. 


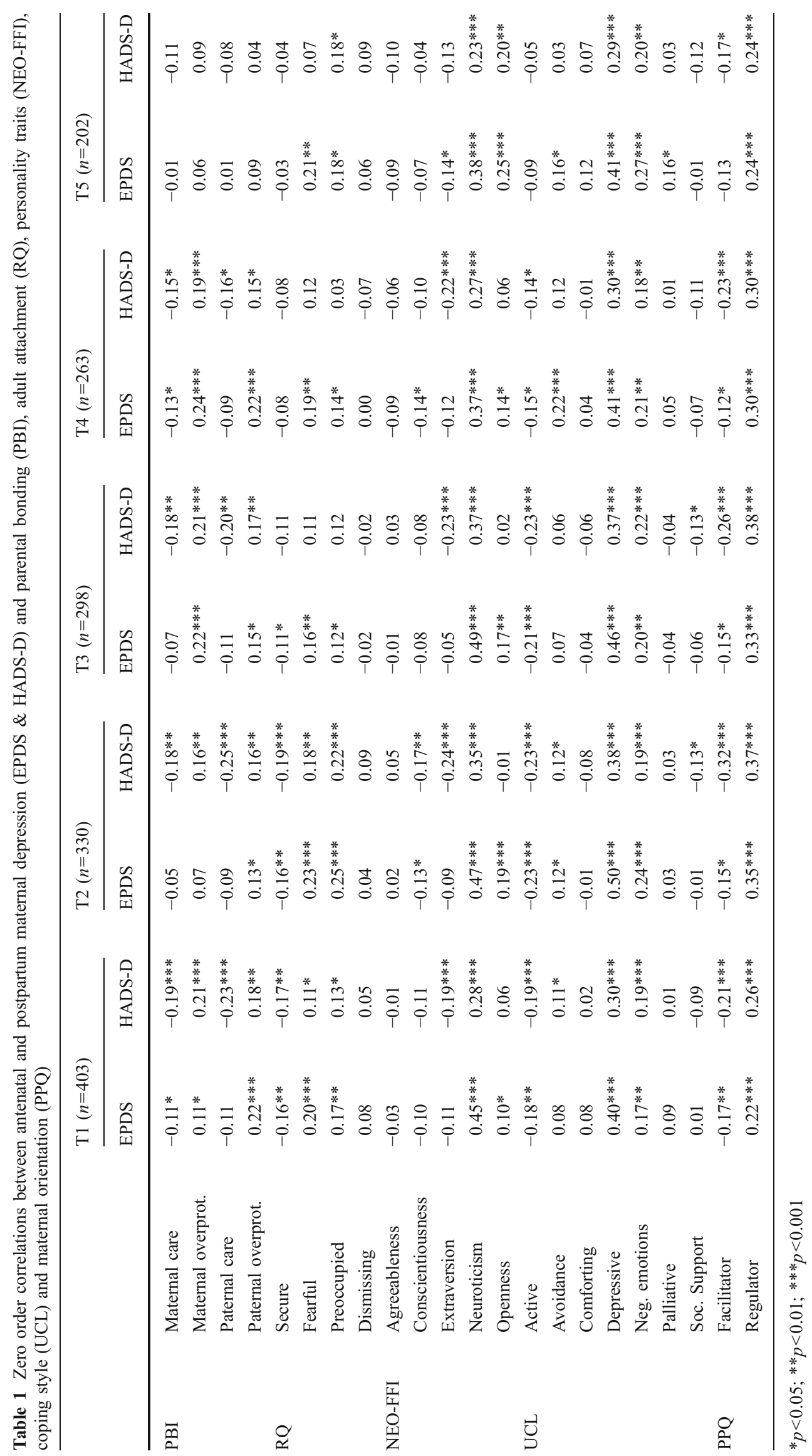




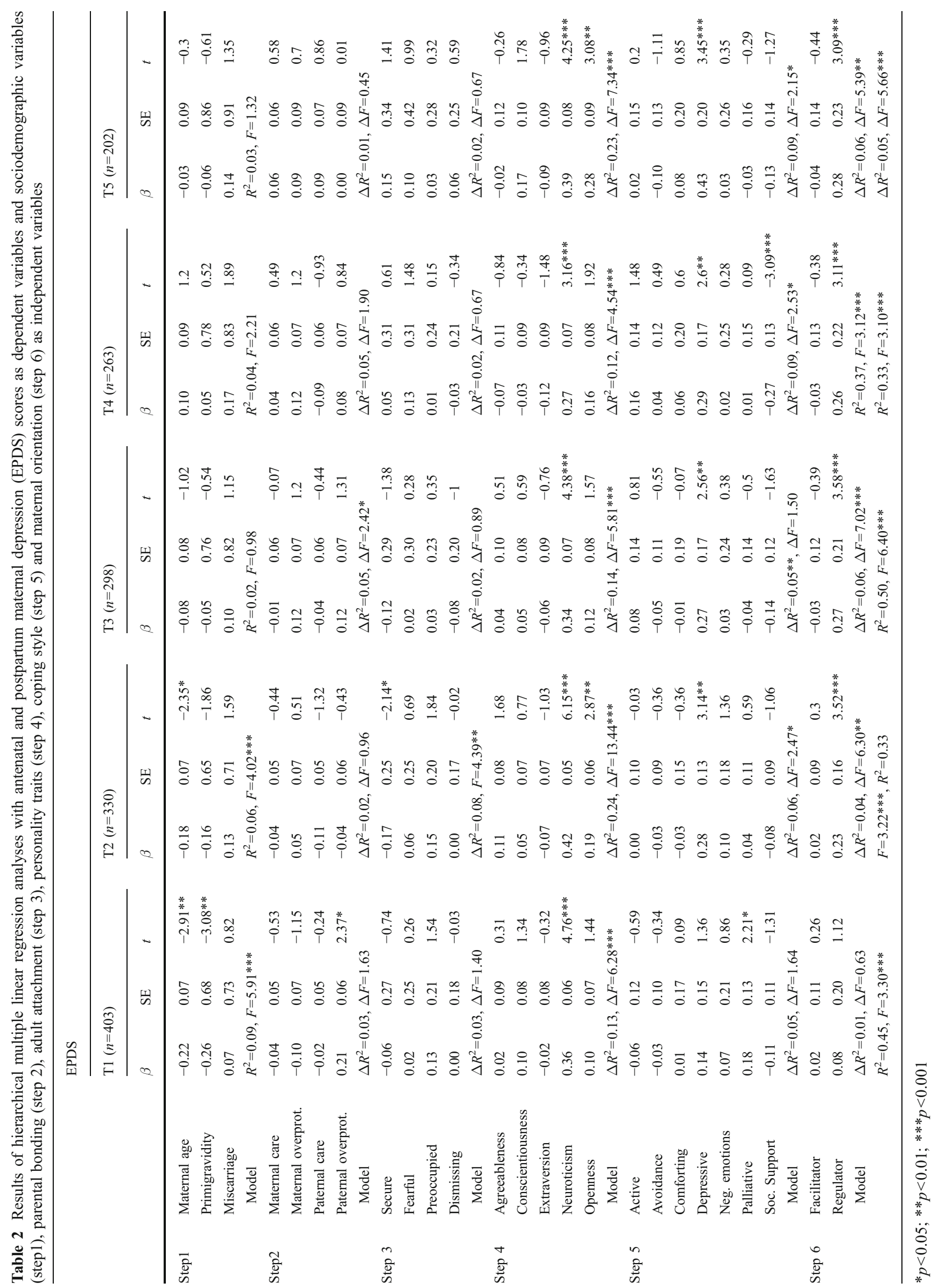




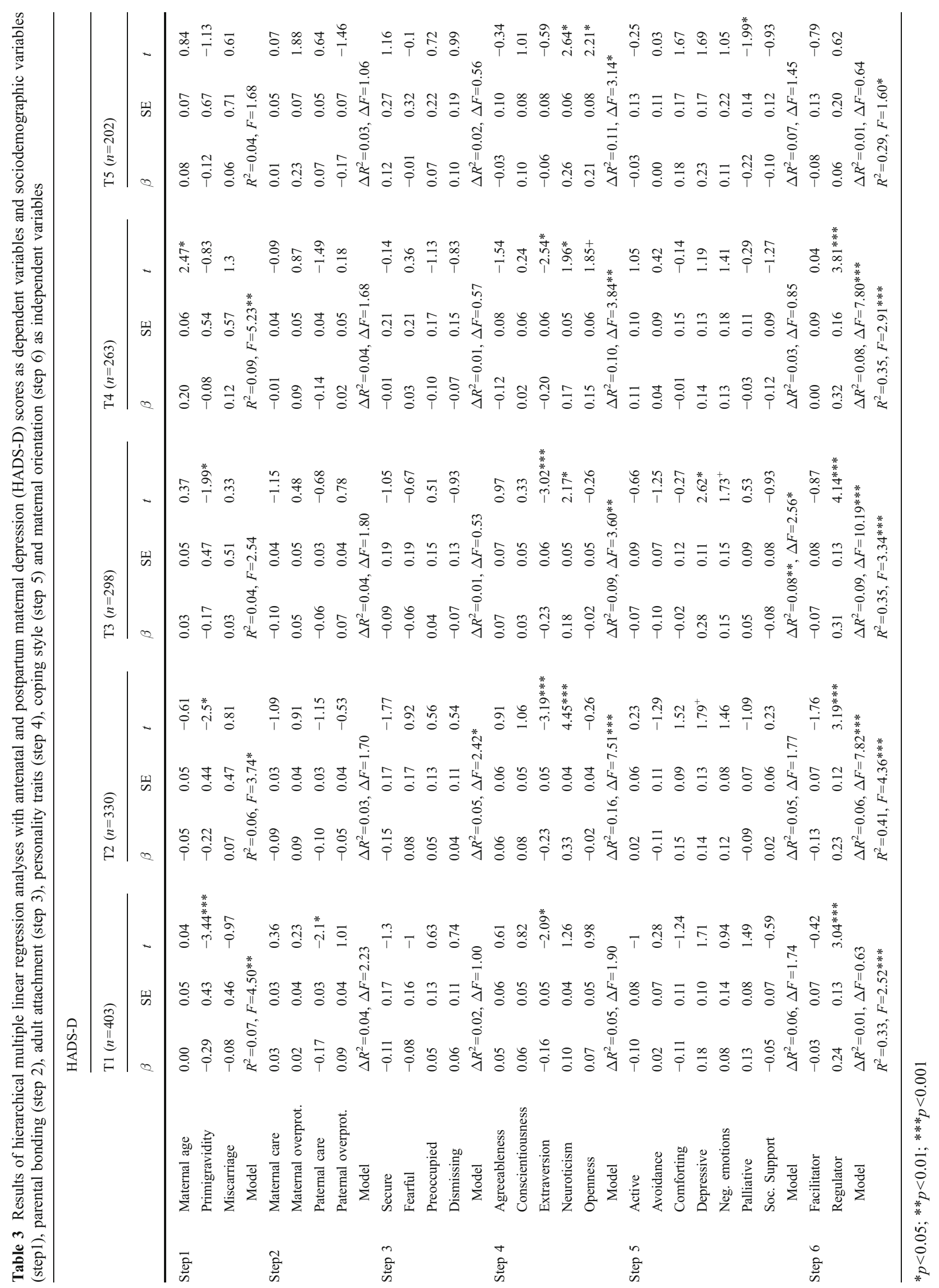


Within these models, higher maternal age was consistently associated with lower scores on the EPDS $\left(\beta_{\mathrm{T} 1}=-0.22\right.$, $\left.\beta_{\mathrm{T} 2}=-0.18\right)$ whereas primigravidity was associated with lower scores on the HADS-D $\left(\beta_{\mathrm{T} 1}=-0.29, \beta_{\mathrm{T} 2}=-0.22\right)$ and EPDS $\left(\beta_{\mathrm{T} 1}=-0.26\right)$. The addition of parental bonding (step 2) contributed significantly but poorly $\left(\Delta R^{2}=0.05, p<0.05\right)$ to the variance of only the EPDS regression model at T3. None of the individual scales, however, were associated with the responses on the EPDS. In the third step, adult attachment was added to the regression models. A significant but small explanatory contribution of this block was found on both the EPDS $\left(\Delta R^{2}=0.08, p<0.01\right)$ and the HADS-D $\left(\Delta R^{2}=0.05, p<0.05\right)$, but only at T2. Higher scores on the secure-attachment scale were negatively associated with the scores on the EPDS $(\beta=-0.17)$ and the HADS-D $(\beta=-0.15)$, although the latter marginally exceeded the level of statistical significance $(p=0.06)$. The contribution of the personality traits (step 4 ) was substantial $\left(\Delta R^{2}\right.$ between 0.12 and 0.24 ) and highly significant (all $p<$ $0.0001)$ in all regression models with the EPDS as dependent variable. Less substantial $\left(\Delta R^{2}\right.$ between 0.05 and 0.16) and less significant contributions were found between T2 and T5 with the HADS-D as dependent variable. Of these personality scales, higher scores on the Neuroticism scale primarily explained higher scores of the EPDS ( $\beta$ between 0.27 and 0.42 ) and the HADS-D ( $\beta$ between 0.17 and 0.33 ). The addition of the block of coping styles (step 5) contributed moderately $\left(\Delta R^{2}\right.$ between 0.05 and 0.9 ) to the variation of the EPDS scores in all but one regression model (T1). A significant and moderate contribution to the variation in HADS-D scores was only found at T3 $\left(\Delta R^{2}=0.08\right)$. Higher scores on the EPDS $(\beta$ between 0.27 and 0.43$)$ and the HADS-D $(\beta=0.28)$ were largely due to higher scores on the depressive reaction scale. Finally, a significant but rather small contribution of the maternal antenatal orientations (step 6) was found on the variance of the responses on the EPDS $\left(\Delta R^{2}\right.$ between 0.01 and 0.09$)$ and the HADS-D $\left(\Delta R^{2}\right.$ between 0.04 and 0.06 ), but not at T1. More specifically, higher scores on the Regulator scale were associated with higher scores on the EPDS ( $\beta$ between 0.23 and 0.32 ) and the HADS-D $(\beta$ between 0.23 and 0.28 ).

\section{HMLR analyses controlled for previous reported EPDS and HADS-D scores}

In a subsequent analysis, the results from the HMLR models were controlled for previous reported EPDS and HADS-D scores. These previous scores contributed substantially $\left(\Delta R^{2}\right.$ between 0.10 and 0.39$)$ and significantly (all $p<0.001$ ) to the variation of the responses on both the EPDS and the HADS-D at T2, T3, T4, and T5. The contribution of the maternal orientations remained sig- nificant at T2, T3, and T4 for both the EPDS and the HADS-D. The extent of the contribution, however, decreased to a marginal level $\left(\Delta R^{2}\right.$ between 0.02 and $0.05)$.

\section{Discussion}

In this study, we investigated the association between the maternal orientations (as described by Raphael-Leff 2005) and the prevalence of depressive symptoms during pregnancy and the postpartum period. In general, the level of depressive symptoms increased in the third pregnancy trimester after it had slightly dropped in the previous trimester. After childbirth, the depressive symptoms decreased at both 8 to 12 weeks (T4) and 20 to 25 weeks (T5) postpartum. Thus, our results concur with those of other large longitudinal studies (Evans et al. 2001; Heron et al. 2004). In addition, we support previous studies identifying antenatal depressive symptoms as one of the strongest predictors of postpartum depressive symptomatology (Robertson et al. 2004).

According to Raphael-Leff's clinical observations, expectant women or new mothers are at risk for psychological distress when they cannot fulfil their own specific expectations about motherhood (Raphael-Leff 2005). For a women tending to the Facilitator orientation, a normal developing pregnancy is expected to be a protective period because the Facilitator feels privileged and enriched to contain, protect, and nourish the foetus. The pregnancy, therefore, contributes to the self-realization and, hence, to the self-esteem of the Facilitator. For the more regulating women, the pregnancy itself will be experienced as intrusive, not something that can be controlled, and a threat to the development of their identities as an adult persons (Raphael-Leff 2005). As a result, pregnant Regulators are thought to express more negative feelings than pregnant Facilitators. In our study, a small protective effect of the Facilitator orientation was found, but only in the bivariate analyses and in the multiple regression analyses with the antenatal HADS-D scores as dependent variable. Within the more complex HMLR models, however, the contribution of the Facilitator orientation was no longer statistically significant. As for the Regulator orientation, our findings support the hypothesis that higher scores on the Regulator scale are associated with higher levels of antenatal depressive symptomatology. This effect remained significant even after controlling for other intrapsychic variables. Moreover, because prior depressive responses could have influenced responses on both the maternal orientation scales and the depression screening instruments, we also controlled for this effect in the regression model. The fact that the effect of the Regulator orientation still remained 
significant affirms its independent contribution to the variance of the reported depressive symptomatology.

For both the Regulator and the Facilitator mother, the first weeks after the delivery are considered to be a vulnerable period (Raphael-Leff 2005). During maternity leave (approximately 3 months in Belgium), the Regulator is inescapably confronted with the constant demands of her child. By day, when her partner is at work, or in the evening, when she is tired because of caring for her child, the Regulator has little time for her own needs. This enforced togetherness and the reduced possibilities of personal development are thought to result in a lowered self-esteem in the Regulator, expressed in depressed feelings (Raphael-Leff 2005; Sharp and Bramwell 2004). Later on, as hypothesized by Raphael-Leff (Raphael-Leff 2005), when the Regulator returns to her pre-pregnancy routine (e.g., employment), these heightened levels of depressive symptoms are hypothesized to decrease. In our study, heightened levels of depressive symptoms in the postpartum period were, indeed, found in women with higher scores on the Regulator scale, even after controlling for the other intrapsychic variables and previously reported depressive symptoms.

Given her idealized views on motherhood and the baby, the Facilitator is expected to be at risk for disappointments in the first weeks after childbirth (Raphael-Leff 2005; Sharp and Bramwell 2004). When mothering is experienced as harder then expected, the Facilitator's self-esteem, based on her wish to be a perfect mother, is thought to decrease. Also, later on, when the Facilitator needs to separate from her child (e.g., to return to employment), these feelings are expected to grow stronger (Raphael-Leff 2005). In our study, higher scores on the Facilitator scale were still significantly but poorly correlated with lower levels of depressive symptoms between 8 and 15 weeks postpartum. Within uncontrolled linear regressions, this association was only confirmed with the HADS-D as a measure of depressive symptomatology. This effect, however, did not remained statistically significant after the other intrapsychic variables and the HADS-D score of T3 were added into the regression model.

The predictive value of Neuroticism for depressive symptomatology is already well established within both a non-clinical population (Jylha and Isometsa 2006) and in a population of pregnant and postpartum women (Robertson et al. 2004). Similarly, a protective effect of the extraversion personality trait against depressive symptomatology was found in the previously mentioned non-clinical population study (Jylha and Isometsa 2006). In line with those findings, the frequent use of depressive, negative, or avoidant coping reactions in pregnant or postpartum women were also found to be positively associated with depressive symptoms and distress (Da Costa et al. 2000;
Demyttenaere et al. 1995; Sanderman and Ormel 1992). Therefore, our results add, to some extent, further evidence to support those findings.

In a non-clinical population, both positive memories of parental attachment during the mother's own childhood and secure adult attachment have been found to be protective against higher levels of depressive symptoms (Carter et al. 2001; Mickelson et al. 1997; Parker 1979a, b; Roberts et al. 1996). In our study, the association between antenatal depressive symptoms and memories of an overprotective mother or father was only weak. A possible explanation lies in the suggestion of Ruble et al. (1990) and Priel and Besser (1999) that the developmental task to develop a selfidentity as a mother is guided with current patterns of attachment, rather than representations based on the past. Thus, not the memories of parental attachment but the actual adult romantic attachment style would then be the primary attachment predictor of the degree of depressive symptoms in pregnant women. In our study, a small correlation was found between a more secure adult attachment style and lower levels of depressive symptoms during the first two pregnancy trimesters (both on the EPDS and HADS-D). However, this relation was small and did not hold within more complex regression models, except for $\mathrm{T} 2$.

As for the postpartum period, it is interesting to note that, based on bivariate analyses, memories of an overprotective mother and father contributed to higher levels of depressive symptoms in the early postpartum period ( 8 to 15 weeks postpartum), both on the EPDS and the HADS-D. Similar results were found by McLaren et al. (2007) and Matthey et al. (2000). One explanation could be that women who were not encouraged by their own mothers to become independent and autonomous adults probably lack the confidence and self-esteem necessary to face the real challenges and responsibilities of motherhood. Nevertheless, one should take into account that our bivariate findings did not hold within the HMLR analyses.

In this study, we opted to include two measures of depressive symptomatology, the EPDS and the HADS-D. As expected, most of our findings with both scales concurred. Nevertheless, some minor differences were found in both the repeated measures analyses and the bivariate and multivariate analyses. These differences are possibly because the HADS-D only addresses depressive symptoms whereas the EPDS also addresses anxiety and panic symptoms (Brouwers et al. 2001). Indeed, the first descriptions of postpartum depression, by Pitt (1968), referred to an atypical form of depressive disorder that was almost invariably accompanied by - and sometimes overshadowed by - anxiety over the child. This specificity, thus, cannot be captured by the depression scale of the HADS.

Several limitations of this study need to be considered. First, women reporting higher levels of depressive symptoms 
during the first pregnancy trimester were more likely to drop out as the study progressed. Although common in mental health research, this phenomenon could have biased the results of this study (Austin et al. 2007; Britton 2007). Also, our data were obtained from a population of subjects who enrolled electively. A selective attrition is possible, given the fact that the participants were well educated and employed. On the other hand, our study population is somewhat representative of both the population of women visiting the antenatal clinic of the UZ Gasthuisberg Leuven and the women living in the region of the hospital. Furthermore, we measured the maternal orientation during the third pregnancy trimester and used it to predict, retrospectively, the degree of depressive symptoms in the first and second pregnancy trimester. Therefore, the interpretation of these results assumes that the maternal orientation is a fairly stable trait over the whole course of the pregnancy, as suggested by Raphael-Leff (2005). Finally, much of the data were based on responses to questionnaires. Although most of them are well validated, an eventual reporting bias is difficult to prevent or estimate. However, given the fact that our results are consistent and correspond with both previous depression theory and research, we believe that our results contribute to a better understanding of depressive symptomatology in pregnant and postpartum women.

\section{Conclusion}

In this study, we found that, in addition to the welldocumented predictors of depressive symptoms in pregnant and postpartum women (neuroticism, extraversion, depressive coping), the model of maternal antenatal orientations, as defined and described by Raphael-Leff (2005), also had a small but significant contribution in the variance of the depressive symptomatology, even after controlling for previously reported depressive symptoms.

Conflict of Interest The authors declare that they do not have a conflict of interest in submitting this manuscript. Johan C. H. van Bussel and Bernard Spitz have nothing to disclose. Koen Demyttenaere has been serving as a member of the advisory board / has been serving as a speaker for Boehringer Ingelheim, Cyberonics, Eli Lilly, Glaxo Smith Kline, Organon, Servier, Lundbeck, and Wyeth. None of these honoraria interfered with the design, the analysis of the data, or the preparation of the manuscript.

\section{References}

Austin MP, Tully L, Parker G (2007) Examining the relationship between antenatal anxiety and postnatal depression. J Affect Disord 101(1-3):169-174

Bartholomew K, Horowitz LM (1991) Attachment styles among young adults: a test of a four-category model. J Pers Soc Psychol 61(2):226-244
Britton JR (2007) Maternal anxiety: course and antecedents during the early postpartum period. Depress Anxiety 25:793-800

Brouwers EP, van Baar AL, Pop VJ (2001) Does the Edinburgh postnatal depression scale measure anxiety? J Psychosom Res 51 (5):659-663

Carter MM, Sbrocco T, Lewis EL, Friedman EK (2001) Parental bonding and anxiety: differences between African American and European American college students. J Anxiety Disord 15 (6):555-569

Chen H, Chan Y, Tan K, Lee T (2004) Depressive symptomatology in pregnancy. Soc Psychiatry Psychiatr Epidemiol 39(12):975-979

Chung TK, Lau TK, Yip AS, Chiu HF, Lee DT (2001) Antepartum depressive symptomatology is associated with adverse obstetric and neonatal outcomes. Psychosom Med 63(5):830-834

Colin C (2006) Robust regression and outliers detection with the ROBUSTREG procedure. SAS Institute, Cary, NC, pp 265-227

Costa P, McCrae R (1990) Personality: another 'hidden factor' in stress research. Psychol Inq 1(1):22-24

Costa P, McCrae R (1992) NEO PI-R. Professional manual. Psychological Assessment Resources, Odessa, FL

Cox JL, Holden JM (1994) Perinatal psychiatry: use and misuse of the Edinburgh Postnatal Depression Scale EPDS. Gaskell, London

Cox JL, Holden JM, Sagovsky R (1987) Detection of postnatal depression. Development of the 10-item Edinburgh Postnatal Depression Scale. Br J Psychiatry 150:782-786

Da Costa D, Larouche J, Dritsa M, Brender W (2000) Psychosocial correlates of prepartum and postpartum depressed mood. J Affect Disord 59(1):31-40

Dayan J, Creveuil C, Herlicoviez M, Herbel C, Baranger E, Savoye C et al (2002) Role of anxiety and depression in the onset of spontaneous preterm labor. Am J Epidemiol 155(4):293-301

Dayan J, Creveuil C, Marks MN, Conroy S, Herlicoviez M, Dreyfus $M$ et al (2006) Prenatal depression, prenatal anxiety, and spontaneous preterm birth: a prospective cohort study among women with early and regular care. Psychosom Med 68(6):938946

Demyttenaere K, Lenaerts H, Nijs P, Van Assche FA (1995) Individual coping style and psychological attitudes during pregnancy and predict depression levels during pregnancy and during postpartum. Acta Psychiatr Scand 91(2):95-102

Evans J, Heron J, Francomb H, Oke S, Golding J (2001) Cohort study of depressed mood during pregnancy and after childbirth. BMJ 323(7307):257-260

Griffin DW, Bartholomew K (1994) The metaphysics of measurement: the case of adult attachment. In: BartholomewK., PerlmanD. (eds) Advances in personal relationships (vol. 5: attachment processes in adulthood). Kingsley, London, pp 17-52

Harwood K, McLean N, Durkin K (2007) First-time mothers' expectations of parenthood: what happens when optimistic expectations are not matched by later experiences? Dev Psychol 43(1):1-12

Heron J, O'Connor TG, Evans J, Golding J, Glover V (2004) The course of anxiety and depression through pregnancy and the postpartum in a community sample. J Affect Disord 80(1):65-73

Herrmann C (1997) International experiences with the Hospital Anxiety and Depression Scale - a review of validation data and clinical results. J Psychosom Res 42(1):17-41

Hoekstra HA, Ormel J, de Fruyt F (1996) Handleiding NEO persoonlijkheids-vragenlijsten NEO-PI-R en NEO-FFI. Swets Test Services, Lisse

Jylha P, Isometsa E (2006) The relationship of neuroticism and extraversion to symptoms of anxiety and depression in the general population. Depress Anxiety 23(5):281-289

Kurki T, Hiilesmaa V, Raitasalo R, Mattila H, Ylikorkala O (2000) Depression and anxiety in early pregnancy and risk for preeclampsia. Obstet Gynecol 95(4):487-490 
Lowyck B, Luyten P, Hutsebaut J, Corveleyn J (2003) De relatievragenlijst van Bartholomew \& Horowitz. Center for Psychoanalysis and Psychodynamic Psychology (K.U.Leuven), Leuven

Matthey S, Barnett B, Ungerer J, Waters B (2000) Paternal and maternal depressed mood during the transition to parenthood. J Affect Disord 60(2):75-85

McCrae RR, Costa PT (1986) Personality, coping, and coping effectiveness in an adult sample. J Person 54(2):385-404

McLaren L, Kuh D, Hardy R, Mishra G (2007) Postnatal depression and the original mother-child relationship: a prospective cohort study. J Affect Disord 100(1-3):211-219

Mickelson KD, Kessler RC, Shaver PR (1997) Adult attachment in a nationally representative sample. J Pers Soc Psychol 73(5):10921106

Misri S, Oberlander TF, Fairbrother N, Carter D, Ryan D, Kuan AJ et al (2004) Relation between prenatal maternal mood and anxiety and neonatal health. Can J Psychiatry 49(10):684-689

Murphy B, Bates GW (1997) Adult attachment style and vulnerability to depression. Pers Individ Differ 22(6):835-844

Murray D, Cox JL (1990) Screening for depression during pregnancy with the Edinburgh Depression Scale (EPDS). J Reprod Infant Psychol 8(2):99-107

Murray G, Rawlings D, Allen N, Trinder J (2003) NEO Five-Factor Inventory Scores: psychometric properties in a community sample. Meas Eval Couns Dev 36(3):140-149

O'Connor TG, Heron J, Glover V (2002) Antenatal anxiety predicts child behavioral/emotional problems independently of postnatal depression. J Am Acad Child Adolesc Psychiatry 41(12):1470-1477

Orr ST, James SA, Blackmore Prince C (2002) Maternal prenatal depressive symptoms and spontaneous preterm births among African-American women in Baltimore, Maryland. Am J Epidemiol 156(9):797-802

Parker G (1979a) Parental characteristics in relation to depressive disorders. Br J Psychiatry 134:138-147

Parker G (1979b) Reported parental characteristics in relation to trait depression and anxiety levels in a non-clinical group. Aust $\mathrm{N} \mathrm{Z} \mathrm{J}$ Psychiatry 13(3):260-264

Parker G (1989) The parental bonding instrument: psychometric properties reviewed. Psychiatr Dev 7(4):317-335

Parker G, Tupling H, Brown L (1979) A parental bonding instrument. J Med Psychol 52:1-10

Perris C, Andersson P (2000) Experiences of parental rearing and patterns of attachment in adulthood. Clin Psychol Psychother 7 (4):279-288

Pitt B (1968) "Atypical” depression following childbirth. $\mathrm{Br} \mathrm{J}$ Psychiatry 114(516):1325-1335

Pop VJ, Komproe IH, van Son MJ (1992) Characteristics of the Edinburgh Post Natal Depression Scale in The Netherlands. J Affect Disord 26(2):105-110

Priel B, Besser A (1999) Vulnerability to postpartum depressive symptomatology: dependency, self-criticism and the moderating role of antenatal attachment. J Soc Clin Psych 18:240-253
Raphael-Leff J (1985) Facilitators and regulators: vulnerability to postnatal disturbance. J Psychosom Obstet Gynaecol 4:151-168

Raphael-Leff J (2005) Psychological processes of childbearing. The Anna Freud Centre, London

Reti IM, Samuels JF, Eaton WW, Bienvenu Iii OJ, Costa PT Jr, Nestadt G (2002) Influences of parenting on normal personality traits. Psychiatry Res 111(1):55-64

Roberts JE, Gotlib IH, Kassel JD (1996) Adult attachment security and symptoms of depression: the mediating roles of dysfunctional attitudes and low self-esteem. J Pers Soc Psychol 70(2):310-320

Robertson E, Grace S, Wallington T, Stewart DE (2004) Antenatal risk factors for postpartum depression: a synthesis of recent literature. Gen Hosp Psychiatry 26(4):289-295

Ruble DN, Brooks-Gunn J, Fleming AS, Fitzmaurice G, Stangor C, Deutsch F (1990) Transition to motherhood and the self: measurement, stability, and change. J Pers Soc Psychol 58(3):450-463

Sanderman R, Ormel J (1992) De Utrechtse Copinglijst. Gedrag en Gezondheid 20:32-37

SAS Institute Inc (2002) SAS software version 9.1 of the SAS system for windows (version 9.1). SAS Institute, Cary, NC

Schreurs P, van de Willige G (1988) Omgaan met problemen en Gebeurtenissen. De Utrechtse Copinglijst. Swets \& Zeitlinger, Lisse

Sharp H, Bramwell R (2004) An empirical evaluation of a psychoanalytic theory of mothering orientation: implications for the antenatal prediction of postnatal depression. J Reprod Infant Psychol 22(2):71-89

Spinhoven P, Ormel J, Sloekers PP, Kempen GI, Speckens AE, Van Hemert AM (1997) A validation study of the Hospital Anxiety and Depression Scale (HADS) in different groups of Dutch subjects. Psychol Med 27(2):363-370

Steer RA, Scholl TO, Hediger ML, Fischer RL (1992) Self-reported depression and negative pregnancy outcomes. J Clin Epidemiol 45(10):1093-1099

Uehara T, Sakado K, Sato T, Someya T (1999) Do perceived parenting styles influence stress coping in patients with major depressive disorders? Stress Med 15(3):197-200

van Bussel JCH, Spitz B, Demyttenaere K (2006) Women's mental health before, during, and after pregnancy: a population-based controlled cohort study. Birth 33(4):297-302

van Bussel JCH, Spitz B, Demyttenaere K (2008) Anxiety in pregnant and postpartum women. An exploratory study of the role of maternal orientations. J Affect Disord 114:232-242 doi:10.1016/ j.jad.2008.07.018

van Bussel JCH, Spitz B, Demyttenaere K (2009) Maternal antenatal orientations on pregnancy, the child and motherhood, reliability and construct validity of a brief measure. Individual Differences Research

Verschueren K, Marcoen A (1993) Gehechtheidsstijl, intimiteit en vertrouwen in de jongvolwassenheid: een reflectie van de ervaren ouderlijke sensitiviteit? Psychol Belg 33:49-76

Zigmond AS, Snaith RP (1983) The hospital anxiety and depression scale. Acta Psychiatr Scand 67(6):361-370 